Proceedings

\title{
Controlled Release of Cinnamon Leaf Oil from Chitosan Microcapsules Embedded within a Sodium Alginate/Gelatin Hydrogel-Like Film for Pseudomonas aeruginosa Elimination ${ }^{+}$
}

\author{
Catarina S. Miranda, Joana C. Antunes, Natália C. Homem and Helena P. Felgueiras *
}

Citation: Miranda, C.S.; Antunes, J.C.; Homem, N.C.; Felgueiras, H.P. Controlled Release of Cinnamon Leaf Oil from Chitosan Microcapsules Embedded within a Sodium Alginate/Gelatin HydrogelLike Film for Pseudomonas aeruginosa Elimination. Proceedings 2021, 69, 39. https://doi.org/10.3390/CGPM202007181

Published: 3 November 2020

Publisher's Note: MDPI stays neutral with regard to jurisdictional claims in published maps and institutional affiliations.

Copyright: $\odot 2020$ by the authors. Licensee MDPI, Basel, Switzerland. This article is an open access article distributed under the terms and conditions of the Creative Commons Attribution (CC BY) license (http://creativecommons.org/licenses/by/4.0/).
Centre for Textile Science and Technology (2C2T), Department of Textile Engineering, University of Minho, Campus of Azurém, 4800-058 Guimarães, Portugal; catarinanda@gmail.com (C.S.M.);

joana.antunes@2c2t.uminho.pt (J.C.A.); natalia.homem@2c2t.uminho.pt (N.C.H.)

* Correspondence: helena.felgueiras@2c2t.uminho.pt; Tel.: +351-253-510-283; Fax: +351-253-510-293

† Presented at the First International Conference on “Green” Polymer Materials 2020, 5-25 November 2020; Available online: https://sciforum.net/conference/CGPM2020.

\begin{abstract}
Pseudomonas aeruginosa is considered a public threat, with antibiotics increasing their resistance. Essential oils (EOs) have demonstrated significant effects against microorganisms. However, due to their volatile nature, they cannot be used in their free-state. Here, hydrogel-like films were produced from a combination of sodium alginate (SA) and gelatin (GN) to serve as delivery platforms for the controlled release of cinnamon leaf oil (CLO) entrapped within chitosan (CS) microcapsules. The minimum inhibitory concentration (MIC) of CLO was established at $39.3 \mathrm{mg} / \mathrm{mL}$ against $P$. aeruginosa. CS microcapsules were prepared via ionotropic gelation with tripolyphosphate (TPP), encapsulating CLO at MIC. Successful production was confirmed by fluorescent microscopy using Nile red as a detection agent. Microcapsules were embedded within a biodegradable SA/GN polymeric matrix processed by solvent casting/phase inversion with SA/GN used at 70/30 polymer ratio at $2 \mathrm{wt}$ \% SA concentration. A concentration of $2 \mathrm{wt} . \% \mathrm{CaCl}_{2}$ was used as a coagulation bath. The CLO-containing CS microcapsules' homogeneous distribution was guaranteed by successive vortex and blending processes applied prior to casting. CLO controlled release from the films was monitored in physiological $\mathrm{pH}$ for $24 \mathrm{~h}$. Hydrated films were obtained, with the presence of loaded CS capsules being confirmed by FTIR. Qualitative/quantitative antimicrobial examinations validated the loaded film potential to fight $P$. aeruginosa.
\end{abstract}

Keywords: bio-based polymers; drug delivery platform; natural extracts; trigger-based release; bactericidal effects

\section{Introduction}

Pseudomonas aeruginosa, a Gram-negative and monoflagellated bacteria is considered a microorganism of great clinical relevance. It is a very powerful opportunistic human pathogen that survives under a great variety of conditions, causing infections, not only in immunocompromised cancer patients but also in patients suffering from burns and cystic fibrosis [1]. P. aeruginosa-derived infections are difficult to eradicate due to this bacterium elevated intrinsic antibiotic resistance. As a result, this microorganism is categorized as a human pathogen with high antibiotic resistance by the Infectious Diseases Society of America [2,3].

Several antimicrobial agents, including antibiotics, have been used to fight $P$. aeruginosa-related infections. Yet, their excessive consumption and misuse has led to an increased appearance of antibiotic resistance [4], hence the current urgency in finding alternatives. In recent years, plant-derived products, possessing promising features, such as antimicrobial, anti-inflammatory and antioxidant effects, have gained a new importance 
as antibiotic replacements by effectively treating infections and causing little impact in the human health [5]. Essential oils (EOs) consist of blends of volatile biomolecules, obtained from different areas of the plants, that defend the host immune system from microorganisms [6]. Many studies have proven their ability to work as alternatives to antibiotics, since they present strong anti-inflammatory and antiseptic properties, for instance [7]. However, their cytotoxicity at elevated concentrations and their high volatility still constitute challenges to overcome prior to their generalized consumption [8].

Polymeric nanocapsules that contain a liquid core encapsulated within a polymerbased shell, have been highlighted as effective drug carriers [9]. By encapsulating antimicrobial agents at the core, these polymeric systems can increase the drug-loading effectiveness, decrease the nanoparticles content and isolate the loaded material from the surrounding environment. Moreover, this isolation prevents secondary undesirable effects related with early degradation and burst release, which can be induced by external factors, like alterations in $\mathrm{pH}$ and temperature, or presence of enzymes [10,11]. The properties of the shell material strongly influence the nanocapsule constancy in a physiological environment, its entrapment effectiveness and loaded molecules release profile. Polysaccharides have been commonly used as transporters of biomolecules, due to their biodegradability rate, compatibility with human cells, gelation capacity and mucoadhesiveness. Furthermore, they possess deprotonated amino or carboxylic acid groups, which will turn the surfaces cationic or anionic, enabling the formation of shells by electrostatic interactions [12]. Chitosan is a natural copolymer, obtained by deacetylation of chitin, that has the ability to form nanocapsules with a positive surface charge. The cationic surface is responsible for improving the interactions between the nanoparticles and some Gram-negative bacteria, through electrostatic interactions, thus increasing its ability to fight infections $[13,14]$.

In this study, an optimized delivery platform was engineered for a targeted and controlled release of cinnamon leaf oil (CLO), a very powerful antimicrobial EO, entrapped within chitosan microcapsules, using hydrogel-like films produced from a combination of sodium alginate (SA) and gelatin (GN). This is the first report on the CLO controlled release from chitosan microcapsules embedded within a SA/GN matrix for the eradication of $P$. aeruginosa bacteria.

Hydrogel-films are widely used for cell microencapsulation since they present a high porosity that results in a high permeability of oxygen, nutrients and metabolites. SA and GN are both very frequently used in biomedicine, due to their biocompatibility and biodegradability [15]. SA is a water-soluble salt and a natural, biocompatible polysaccharide found in brown algae [1,2] endowed with great biocompatibility, low immunogenicity and minor gelation conditions [14]. SA is often used in cell encapsulation since it presents a fast ionic gelation with divalent cations [15]. However, alginate alone is unable to provide a good cell attachment, which will result in low interactions between the cells and the material. In addition, this polymer displays a random degradation profile [16]. Therefore, in order to overcome these limitations, GN is frequently applied $[17,18]$. GN is a protein, formed by collagen hydrolysis, and has been used for controlled drug release due to its biodegradability and biocompatibility [19].

\section{Experiments}

\subsection{Materials}

Pure cinnamon leaf oil (CLO, extracted from Cinnamomum zeylanicum, $\rho=1.049$ ) was obtained from Folha d'Água Company (Santo Tirso, Portugal). Nutrient broth (NB) and nutrient agar (NA) were purchased from VWR, while Mueller-Hinton broth (MHB) was acquired from CondaLab. P. aeruginosa (ATCC 25853) was obtained from the American Type Culture Collection (ATCC).

Chitosan (CS, Mw 100-300 kDa, Acros Organics, Fair Lawn, NJ, USA) and sodium tripolyphosphate (TPP, Sigma, St. Louis, MO, USA) were used in the production of the 
microcapsules. Gelatin (GN, 300 Bloom, Type A from porcine skin) and sodium alginate (alginic acid sodium salt, SA, from brown algae, $\geq 2000 \mathrm{cP}$ ) were acquired from Sigma and used to produce hydrogel-like films. Distilled water $\left(\mathrm{dH}_{2} \mathrm{O}\right)$ was used as the polymer's solvents, and a 2-wt.\% solution of calcium chloride $\left(\mathrm{CaCl}_{2}\right.$ anhydrous, $\left.\mathrm{Chem}-\mathrm{Lab}\right)$ was employed as a coagulation bath.

\subsection{Scanning Electron Microscopy (SEM) Observations}

Visual studies using SEM were carried out to evaluate the potential of CLO to affect the morphology of the $P$. aeruginosa bacteria. Bacteria suspensions were prepared with a concentration of $1 \times 10^{7} \mathrm{CFUs} / \mathrm{mL}$ in $\mathrm{MHB}$, and combined with CLO at minimum inhibitory concentration (MIC) $(39.3 \mathrm{mg} / \mathrm{mL})$, at a $50 / 50 \%$ (v/v) [20]. Afterwards, $500 \mu \mathrm{L}$ of the solution (composed of CLO and bacteria) were put into contact with 12-well tissue culture plates (TCPS) and left at $37^{\circ} \mathrm{C}$ for $24 \mathrm{~h}$, with stirring at $120 \mathrm{rpm}$. The control consisted of bacteria free from the influence of the oil. Then, the media was eliminated, and surfaces were treated with $500 \mu \mathrm{L}$ of $2.5 \%(\mathrm{v} / \mathrm{v})$ of glutaraldehyde in PBS for $1 \mathrm{~h}$ at room temperature (RT), to fix the cells in their current form. Finally, the plates were washed with $\mathrm{dH}_{2} \mathrm{O}$ and using ethanol dilutions $(55,70,80,90,95,100 \%(\mathrm{v} / \mathrm{v}), 30 \mathrm{~min}$ each at RT) the cells were subsequently dehydrated. In the end, the residual ethanol was evaporated at RT. Samples were collected from the TCPS plates using a hot press-on device and covered with a 10-mm thickness film of $\mathrm{Au}-\mathrm{Pd}(80-20 \mathrm{wt} . \%)$ in a 208-resolution sputter coater (Cressington Company, Watford, UK), with an MTM-20 Cressington high resolution thickness controller. Lastly, bacteria were observed via FEG-SEM (NOVA 200 Nano SEM, FEI Company, Montreal, QC, Canada), with an electron accelerating voltage of $10 \mathrm{kV}$.

\subsection{CLO Encapsulation: Chitosan Microcapsules Production}

Prior to any processing, CS was desiccated at $50{ }^{\circ} \mathrm{C}$ for $24 \mathrm{~h}$ to eliminate any water trace. For microcapsule production, four processing conditions were selected (Table 1 ). In all cases, firstly, $\mathrm{CS}$ was hydrated in $\mathrm{dH}_{2} \mathrm{O}$ for $30 \mathrm{~min}$, and then acetic acid was added dropwise (under strong stirring), and left blending for $2 \mathrm{~h}$. TPP was prepared in $\mathrm{dH}_{2} \mathrm{O}$ under slow stirring for $1 \mathrm{~h}$. In case of the CS4 and CS4N solutions, the $\mathrm{pH}$ of both CS and TPP was set to 5.0.

CLO was added dropwise to the CS1 and CS4 and left to blend for $10 \mathrm{~min}$. In the CS1N and CS4N (Nile Red-containing samples), CLO corresponding volumes were also added, and 2 min after its addition, a solution containing Nile Red was added and left under stirring for $8 \mathrm{~min}$. Then, TPP was combined dropwise with all solutions under strong stirring. The solutions were homogenized in an Ultraturrax (T25 digital, IKA ${ }^{\circledR}$ ) on a pulsation mode (10 s ON, $10 \mathrm{~s}$ OFF) repeated 5 times, and then transferred to dialysis membranes (SnakeSkin 10 kDa cut-off, Thermo Fisher, Waltham, MA, USA). The dialysis was performed in $\mathrm{dH}_{2} \mathrm{O}$ and the water was exchanged 3 times over a period of $24 \mathrm{~h}$.

Table 1. Chitosan (CS) microcapsules processing conditions (* after $\mathrm{pH}$ adjustment).

\begin{tabular}{|c|c|c|c|c|c|c|c|c|c|}
\hline Sample & CS (mg) & $\mathrm{dH}_{2} \mathrm{O}(\mathrm{mL})$ & Acetic Acid $(\mu \mathrm{L})$ & CLO $(\mu \mathrm{L})$ & Nile Red $(\mu \mathrm{L})$ & TPP (mL) & pH Adjust & $\mathrm{NaOH}(\mathrm{mL})$ & $\mathrm{dH}_{2} \mathrm{O} *(\mathrm{~mL})$ \\
\hline CS1 & 2.5 & 9.9 & 100 & 834 & - & 9 & No & - & - \\
\hline CS1N & 2.5 & 9.9 & 100 & 834 & 166 & 9 & No & - & - \\
\hline CS4 & 2.5 & 6.9 & 100 & 834 & - & 9 & Yes & 1.1 & 1.9 \\
\hline CS4N & 2.5 & 6.9 & 100 & 834 & 166 & 9 & Yes & 1.1 & 1.9 \\
\hline
\end{tabular}

\subsection{SA/GN Films Production}

SA/GN films were produced by mixing the SA and GN solutions in a ratio of 70:30 $\mathrm{v} / \mathrm{v}$. SA $(2 \% \mathrm{w} / \mathrm{v})$ and $\mathrm{GN}(\sim 1 \% \mathrm{w} / \mathrm{v})$ were dissolved separately in $\mathrm{dH}_{2} \mathrm{O}$ at $50{ }^{\circ} \mathrm{C}$ under stirring for $3 \mathrm{~h}$ and $1 \mathrm{~h}$, respectively. GN was added to the SA solution and blended under slow stirring until a homogeneous solution was obtained $(\sim 1 \mathrm{~h})$. The solutions were then poured into glass petri dishes of $140 \mathrm{~mm}$ diameter $(40 \mathrm{~mL})$, left at $4{ }^{\circ} \mathrm{C}$ overnight to remove 
air bubbles, and subsequently slowly dried at RT for 6 days. A 2 wt. $\% \mathrm{CaCl}_{2}$ solution was then added to the petri dishes containing the films for crosslinking. After $1 \mathrm{~h}$ of contact, the films were detached from the dishes and washed in $100 \mathrm{~mL}$ of $\mathrm{dH}_{2} \mathrm{O}$ at $150 \mathrm{rpm}$ for 5 min, a process that was repeated 3 times. To prepare the SA/GN films containing CLO microcapsules, the solutions CS1 and CS4, after being subjected to dialysis, were added to the SA/GN solutions and kept under low stirring for $30 \mathrm{~min}$; their water content was used as complementing solvent for the polymers. The following steps were kept equal to the unloaded films.

\subsection{Chemical Characterization: ATR-FTIR}

Attenuated total reflectance-Fourier transform infrared spectroscopy (ATR-FTIR) was used to analyze the surface and structural chemistry of the films. The wavenumber ranged between 400 and $4000 \mathrm{~cm}^{-1}$ using an IRAffinity-1S (Shimadzu, Japan), coupled with a diamond crystal. A total of 200 scans were conducted per film type at a $2-\mathrm{cm}^{-1}$ spectral resolution.

\subsection{CLO Release Profile from the Loaded Films}

Unloaded and loaded films were left at $37^{\circ} \mathrm{C}$ and $120 \mathrm{rpm}$ in simulated body fluid (SBF) prepared at physiological $\mathrm{pH}$ (7.4). The release of CLO was monitored after 1, 2, 6 and $24 \mathrm{~h}$ of incubation. A spectophotometer UV-VIS (UV-1800, Shimadzu, Kyoto, Japan) was used to analyze the CLO presence within the SBF (release) overtime at the wavelength of $280 \mathrm{~nm}$ (characteristic of CLO) [21].

\subsection{Time-Kill Kinetics}

$P$. aeruginosa was blended with $\mathrm{NB}$ at $1 \times 10^{5} \mathrm{CFUs} / \mathrm{mL}$, and then combined with CLO or CLO-loaded films at MIC. Free bacteria and unmodified SA/GN films were used as the control. Testing cultures were incubated at $37^{\circ} \mathrm{C}$ and $120 \mathrm{rpm}$, and after 1, 2, 6 and $24 \mathrm{~h}$, aliquots were collected and diluted in PBS $\left(10^{1}\right.$ to $\left.10^{5}\right)$ for further culturing in NA plates for $24 \mathrm{~h}$ at $37^{\circ} \mathrm{C}$. Live colonies were identified and counted, and the resulting amount expressed in mean \pm standard deviation (S.D.). Log reduction studies were performed between unloaded and loaded films and reported as the mean \pm S.D $(n=3$, GraphPad Prism 7.0 software).

\section{Results and Discussion}

\subsection{Cell Wall Disruption}

The CLO interference with the Gram-negative bacteria cell membrane was examined via SEM after $24 \mathrm{~h}$ of contact with CLO at MIC. Figure 1 shows the resulting morphology of the bacteria. Prior to any contact with the antimicrobial agent, $P$. aeruginosa bacteria exhibited a rod-like shape structure with a very smooth and uninterrupted surface. $P$. aeruginosa cells may also present polar flagella that confer the cells with mobility [20,22]. Here, however, those were not detected. Still, many cells revealed signs of undergoing polar binary fission.

CLO acts against the cytoplasmic membrane of bacteria by accumulating at the cell surface and disrupting its functions and integrity, increasing the odds of cell content leakage and ultimately cell death [23]. Here, it was evident that CLO increased the P. aeruginosa bacteria permeability, deforming its original morphology with the appearance of holes or wrinkles along the membrane. CLO surrounded the cells, isolating them for an effective permeabilization. Since these bacteria are relatively more resistant to hydrophobic biomolecules than Gram-positive bacteria to overcome their impermeability, CLO relied on the isolation of cells, in order to traverse the outer cell layer and reach the intracellular space $[20,24]$. 


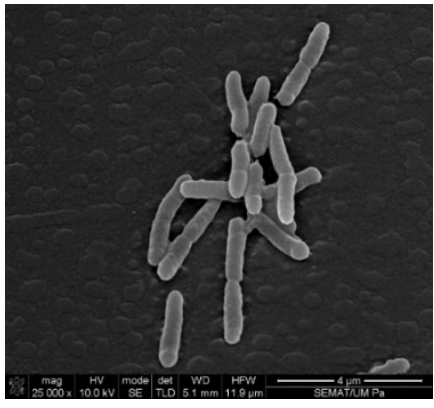

(a)

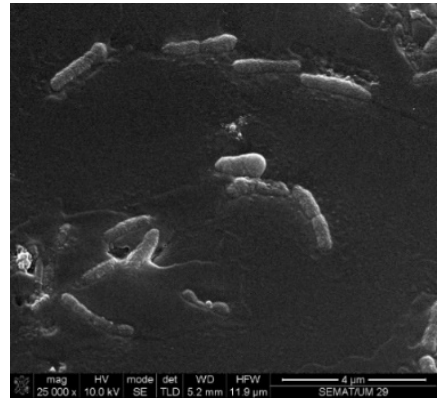

(b)

Figure 1. Micrographs of the P. aeruginosa morphology: (a) before and (b) after contact with cinnamon leaf oil (CLO).

\subsection{Microcapsules Observation}

Fluorescence Microscopy (DM IL LED, Leica Mycrosystems, Wetzlar, Germany) with 40× magnification was applied to observe the microcapsules. Figure 2 represents the morphology of CS1 and CS4 samples before and after dialysis, prior to their incorporation onto films and to any contact with $P$. aeruginosa. For both samples (CS1 and CS4) it was evident that the non-reacted polymer was eradicated after dialysis (Figure 2b,d). Furthermore, it is visible that the sample CS4 contains larger microcapsules and more unloaded chitosan aggregation (Figure 2c,d) than CS1. Therefore, it was concluded that the production of the samples without $\mathrm{pH}$ adjustment (CS1) was more effective.

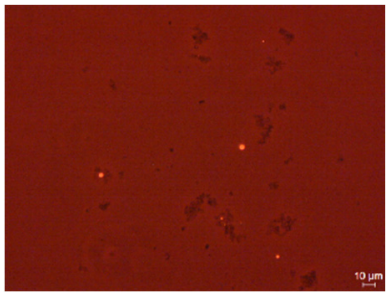

(a)

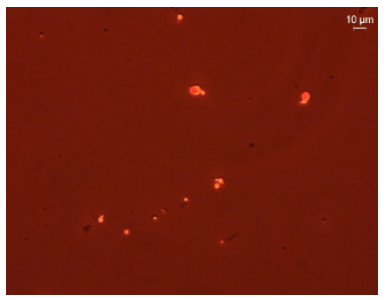

(b)

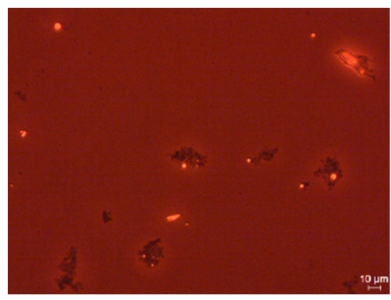

(c)

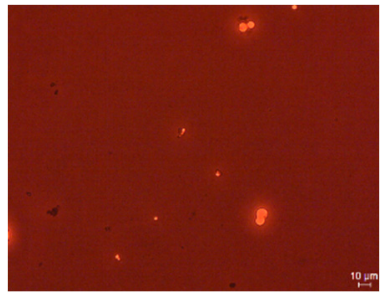

(d)

Figure 2. CS1 and CS4 morphology $(\mathbf{a}, \mathbf{c})$ before and $(\mathbf{b}, \mathbf{d})$ after dialysis, respectively.

\subsection{Chemical Characterization}

ATR-FTIR spectra of the SA/GN films unloaded with loaded with CS1 and CS4 were collected (Figure 3). A slightly broad band centered at $\approx 3420 \mathrm{~cm}^{-1}$ was observed in all spectra and it was attributed to the hydrogen bonded $\mathrm{O}-\mathrm{H}$ stretching vibrations. It was explained by the polymers' affinity to water molecules. Characteristic peaks of each individual polymer were detected at 1416 and $1036 \mathrm{~cm}^{-1}$ for SA, ascribed to the symmetric stretching vibration of the carboxylate group and the $\mathrm{C}-\mathrm{O}$ stretching vibrations [15], respectively, and at 1225 and $1063 \mathrm{~cm}^{-1}$ for GN, which revealed the presence of amide III (stretching bonds of $\mathrm{C}-\mathrm{H}$ and bending bonds of $\mathrm{N}-\mathrm{H}$ ) and $\mathrm{C}-\mathrm{O}$ stretching vibrations $[17,18]$, respectively. Presence of CLO was detected by the appearance of a peak at $1724 \mathrm{~cm}^{-1}$, appointed to the $\mathrm{C}=\mathrm{O}$ stretching vibrations of the oil components, and the presence of a very smooth peak at $1543 \mathrm{~cm}^{-1}$, which is attributed to the aromatic ring $\mathrm{C}=\mathrm{C}$ skeleton vibration [4]. CLO was more easily noticeable on the CS4-containing films than on the CS1, which may indicate that the $\mathrm{EO}$ molecules were larger. 


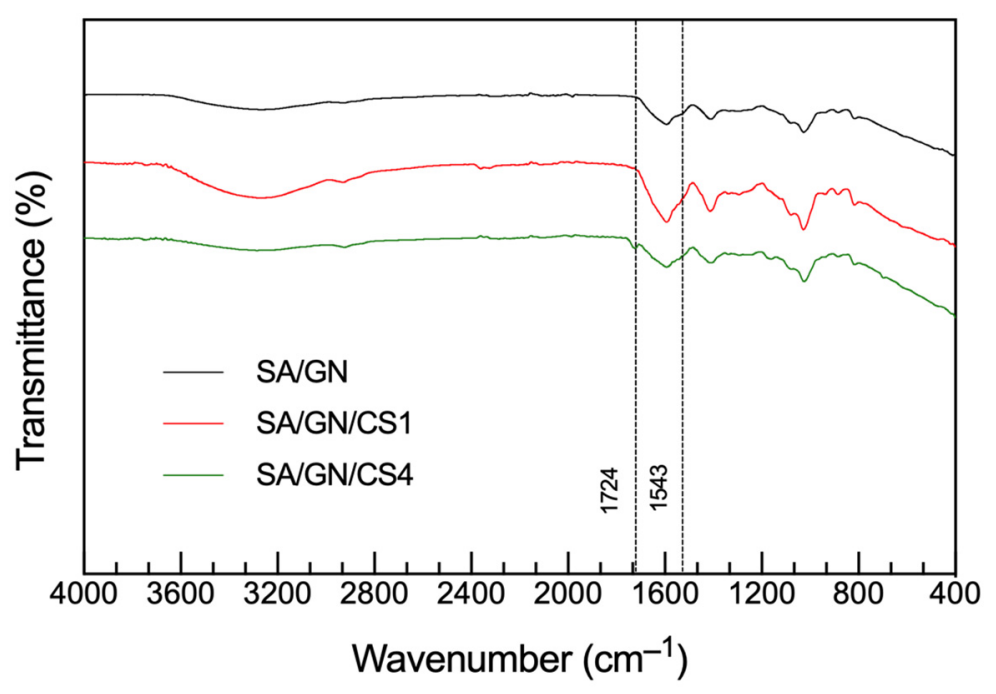

Figure 3. Attenuated total reflectance (ATR)-FTIR spectra of the sodium alginate (SA)/gelatin (GN) unloaded and loaded with CS1 and CS4.

\subsection{CLO Release Profile}

The CLO release profile from the CS1 and CS4 loaded films in the presence of SBF was assessed for a period of $24 \mathrm{~h}$ (Figure 4). The influence of SA and GN was considered and eliminated from the obtained profile. SA/GN/CS1 released the CLO gradually, in a constant-like manner, with the strongest burst release being detected at the 2-h period and reaching an equilibrium within the 24-h period. On the other hand, the SA/GN/CS4 samples, were only able to start releasing the CLO after $2 \mathrm{~h}$. For the entire test, their release profile was always inferior to that of the CS1-containing films. This occurred because when the CS and TPP $\mathrm{pH}$ 's were adjusted to 5, a stronger interface was achieved between the capsules and the films, thus narrowing CLO's release. Moreover, at this $\mathrm{pH}$ the higher presence of clusters (as observed in Figure 2) may have reflected in a greater oil retention under the tested conditions.

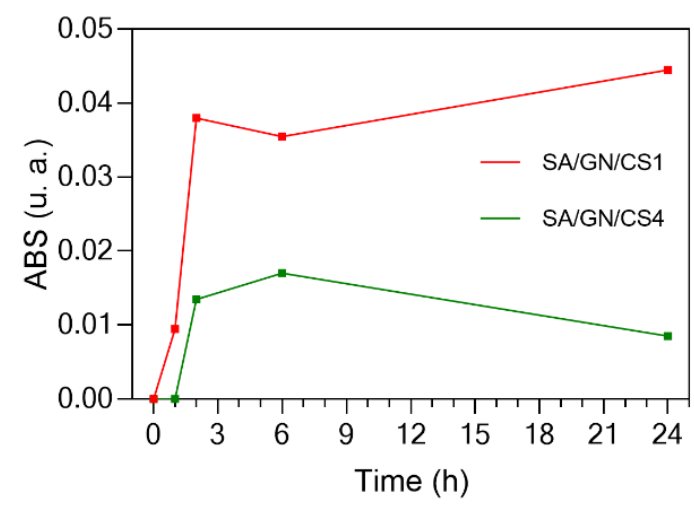

Figure 4. CLO release profile from $1 \mathrm{~h}$ to $24 \mathrm{~h}$ of incubation in simulated body fluid (SBF).

\subsection{Time-Kill Kinetics}

The time-kill curves of the loaded films were assessed by the number of live cells after 1, 2, 6 and $24 \mathrm{~h}$ (Figure 5). The relative $\log$ reduction between the unloaded and loaded films was also verified. In the first hour of interaction, all films promoted the bacteria growth. However, as the release of the CLO from the films intensified (Figure 4), so did its action against the bacterium. Indeed, at the 2-h target the SA/GN/CS1 experienced a significant reduction in the number of viable bacteria. Furthermore, its log reduction became significantly enhanced in comparison to the CS4-loaded films. From $6 \mathrm{~h}$ to $24 \mathrm{~h}$ 
the reduction in viable cells continued. The main component of CLO, the eugenol, is known to interfere with the intracellular roles or ion transport of the cells, causing difficulty for metabolites and connection pathways from taking place and resulting in cell death [4]. Still, due to the reduced release of the CS4 from the films after $6 \mathrm{~h}$, the action against the bacterium became compromised on the CS4-containing films, allowing viable cells to continue multiplying and increasing their number in the media. Once again, the presence of agglomerates that surrounded the CLO-loaded microcapsules may have prevented their release and consequent effective action.
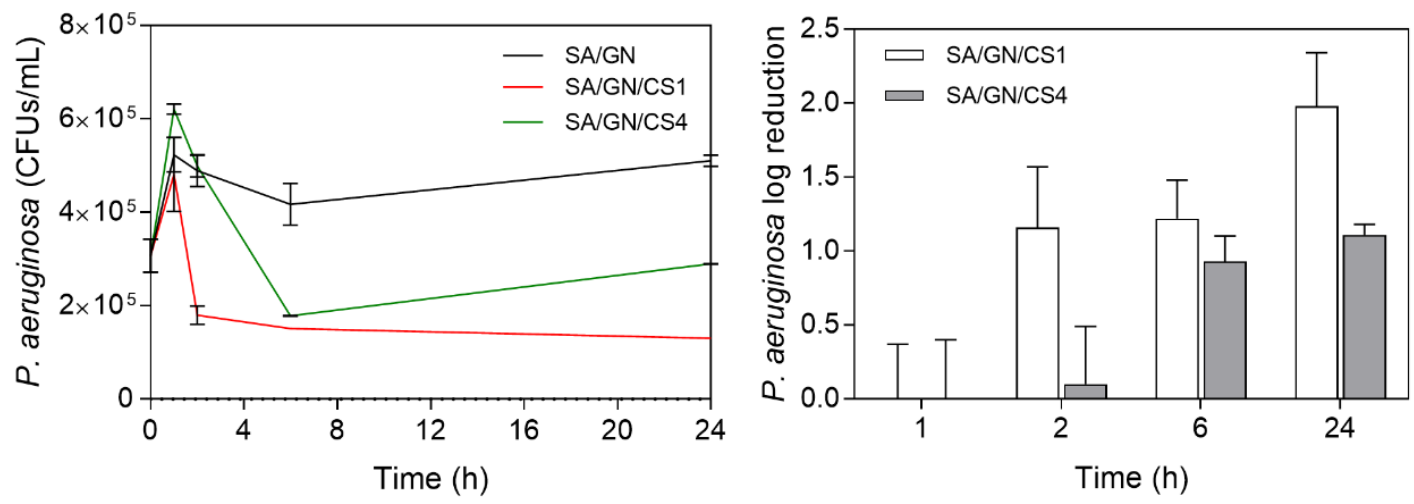

Figure 5. Time-kill kinetics of the P. aeruginosa bacteria in contact with the unloaded and loaded films and log reduction in the loaded films in relation to the control (unloaded SA/GN).

\section{Conclusions}

At the end of this research, it was confirmed the incorporation of the CLO-containing CS microcapsules within the SA/GN fibers. The continuous release of the entrapped oil over a period of $24 \mathrm{~h}$ was attained, with matched time-kill kinetics against the $P$. aeruginosa bacteria. CS1 loaded films were determined more effective than the CS4-loaded or the unloaded surfaces. Future work will be aimed at improving the loading capacity and homogeneity of the microcapsules, so a more effective antimicrobial profile and prolonged release can be achieved.

Author Contributions: conceptualization, C.S.M., J.C.A., N.C.H.; writing original draft, C.S.M.; revision and editing, J.C.A., N.C.H., H.P.F.; supervision, N.C.H., H.P.F.; funding acquisition, H.P.F. All authors have read and agreed to the published version of the manuscript.

Institutional Review Board Statement: Not applicable.

Informed Consent Statement: Not applicable.

Data Availability Statement: Not applicable.

Acknowledgments: Authors acknowledge the Portuguese Foundation for Science and Technology.

Conflicts of Interest: The authors declare no conflict of interest.

\section{References}

1. Wu, W.; Jin, Y. Pseudomonas aeruginosa; Elsevier Ltd.: Amsterdam, The Netherlands, 2014, doi:10.1016/B978-0-12-397169-2.00041$X$.

2. Buhl, M.; Peter, S.; Willmann, M. Prevalence and risk factors associated with colonization and infection of extensively drugresistant Pseudomonas aeruginosa: A systematic review. Expert Rev. Anti-Infect. Ther. 2015, 13, 1159-1170, doi:10.1586/14787210.2015.1064310.

3. Sader, H.S.; Jones, R.N. Antimicrobial susceptibility of uncommonly isolated non-enteric Gram-negative bacilli. Int. J. Antimicrob. Agents. 2005, 25, 95-109, doi:10.1016/j.ijantimicag.2004.10.002.

4. Felgueiras, H.P.; Homem, N.C.; Teixeira, M.A.; Ribeiro, A.R.M.; Antunes, J.C.; Amorim, M.T.P. Physical, thermal, and antibacterial effects of active essential oils with potential for biomedical applications loaded onto cellulose acetate/polycaprolactone wet-spun microfibers. Biomolecules 2020, 10, 1-20, doi:10.3390/biom10081129. 
5. MacLean, R.C.; Millan, A.S. The evolution of antibiotic resistance. Science 2019, 365, 1082-1083, doi:10.1126/science.aax3879.

6. Tavares, T.D.; Antunes, J.C.; Ferreira, F.; Felgueiras, H.P. Biofunctionalization of Natural Fiber-Reinforced Biocomposites for Biomedical Applications. Biomolecules 2020, 10, 148, doi:10.3390/biom10010148.

7. Omonijo, F.A.; Ni, L.; Gong, J.; Wang, Q.; Lahaye, L.; Yang,C. Essential oils as alternatives to antibiotics in swine production. Anim. Nutr. 2018, 4, 126-136, doi:10.1016/j.aninu.2017.09.001.

8. Miranda, C.S.; Ribeiro, A.R.M.; Homem, N.C.; Felgueiras, H.P. Spun Biotextiles in Tissue Engineering and Biomolecules Delivery Systems. Antibiotics 2020, 9, 174, doi:10.3390/antibiotics9040174.

9. Deng, S.; Gigliobianco, M.R.; Censi, R.; Di Martino, P. Polymeric nanocapsules as nanotechnological alternative for drug delivery system: Current status, challenges and opportunities. Nanomaterials 2020, 10, 847, doi:10.3390/nano10050847.

10. Frank, L.A.; Gazzi, R.P.; de Andrade Mello, P.; Buffon, A.; Pohlmann, A.R.; Guterres, S.S. Imiquimod-loaded nanocapsules improve cytotoxicity in cervical cancer cell line. Eur. J. Pharm. Biopharm. 2019, 136, 9-17, doi:10.1016/j.ejpb.2019.01.001.

11. Raffin Pohlmann, A.; Weiss, V.; Mertins, O.; Pesce da Silveira, N.; Stanisçuaski Guterres, S. Spray-dried indomethacin-loaded polyester nanocapsules and nanospheres: Development, stability evaluation and nanostructure models. Eur. J. Pharm. Sci. 2002, 16, 305-312, doi:10.1016/S0928-0987(02)00127-6.

12. Chen, H.; Wang, L.; Yeh, J.; Wu, X.; Cao, Z.; Wang, Y.A.; Zhang, M.; Yang, L.; Mao, H. Reducing non-specific binding and uptake of nanoparticles and improving cell targeting with an antifouling PEO-b-P $\gamma$ MPS copolymer coating. Biomaterials 2010, 31, 53975407, doi:10.1016/j.biomaterials.2010.03.036.

13. Jeon, S.J.; Oh, M.; Yeo, W.S.; Galvão, K.N.; Jeong, K.C. Underlying mechanism of antimicrobial activity of chitosan microparticles and implications for the treatment of infectious diseases. PLoS ONE 2014, 9, e92723, doi:10.1371/journal.pone.0092723.

14. Benhabiles, M.S.; Salah, R.; Lounici, H.; Drouiche, N.; Goosen, M.F.A. ; Mameri, N. Antibacterial activity of chitin, chitosan and its oligomers prepared from shrimp shell waste. Food Hydrocoll. 2012, 29, 48-56, doi:10.1016/j.foodhyd.2012.02.013.

15. Sarker, B.; Papageorgiou, D.G.; Silva, R.; Zehnder, T.; Gul-E-Noor, F.; Bertmer, M.; Kaschta, J.; Chrissafis, K.; Detsch, R.; Boccaccini, A.R. Fabrication of alginate-gelatin crosslinked hydrogel microcapsules and evaluation of the microstructure and physico-chemical properties. J. Mater. Chem. B. 2014, 2, 1470-1482, doi:10.1039/c3tb21509a.

16. Boontheekul, T.; Kong, H.J.; Mooney, D.J. Controlling alginate gel degradation utilizing partial oxidation and bimodal molecular weight distribution. Biomaterials 2005, 26, 2455-2465, doi:10.1016/j.biomaterials.2004.06.044.

17. Balakrishnan, B.; Jayakrishnan, A. Self-cross-linking biopolymers as injectable in situ forming biodegradable scaffolds. Biomaterials 2005, 26, 3941-3951, doi:10.1016/j.biomaterials.2004.10.005.

18. Bigi, A.; Panzavolta, S.; Rubini, K. Relationship between triple-helix content and mechanical properties of gelatin films. Biomaterials 2004, 25, 5675-5680, doi:10.1016/j.biomaterials.2004.01.033.

19. Dong, Z.; Wang, Q.; Du, Y. Alginate/gelatin blend films and their properties for drug controlled release. J. Memb. Sci. 2006, 280, 37-44, doi:10.1016/j.memsci.2006.01.002.

20. Tavares, T.D.; Antunes, J.C.; Padrão, J.; Ribeiro, A.I.; Zille, A.; Amorim, M.T.P.; Ferreira, F.; Felgueiras, H.P. Activity of specialized biomolecules against gram-positive and gram-negative bacteria. Antibiotics 2020, 9, 314, doi:10.3390/antibiotics9060314.

21. Carson, C.F.; Mee, B.J.; Riley, T.V. Mechanism of action of Melaleuca alternifolia (tea tree) oil on Staphylococcus aureus determined by time-kill, leakage, and salt tolerance assays and electron microscopy. Antimicrob. Agents Chemother. 2002, 46, 1914-1920, doi:10.1128/AAC.46.6.1914.

22. Hill, L.E.; Gomes, C.; Taylor, T.M. Characterization of beta-cyclodextrin inclusion complexes containing essential oils (transcinnamaldehyde, eugenol, cinnamon bark, and clove bud extracts) for antimicrobial delivery applications. LWT Food Sci. Technol. 2013, 51, 86-93, doi:10.1016/j.lwt.2012.11.011.

23. Kirisits, M.J.; Prost, L.; Parsek, M.R.; Characterization of Colony Morphology Variants Isolated from. Am. Soc. Microbiol. 2005, 71, 4809-4821, doi:10.1128/AEM.71.8.4809.

24. Nazzaro, F.; Fratianni, F.; De Martino, L.; Coppola, R.; De Feo, V. Effect of essential oils on pathogenic bacteria. Pharmaceuticals 2013, 6, 1451-1474, doi:10.3390/ph6121451. 